\title{
Solid Particles Injection in Gas Turbulent Channel Flow
}

\author{
Abd Elnaby Kabeel, Medhat Elkelawy*, Hagar Alm-Eldin Bastawissi, Ahmed Mohammed Elbanna \\ Mechanical Power Engineering Department, Faculty of Engineering, Tanta University, Tanta, Egypt \\ Email: ^medhat_abo@yahoo.com, *medhatelkelawy@f-eng.tanta.edu.eg
}

How to cite this paper: Kabeel, A.E., Elkelawy, M., Bastawissi, H.A.-E. and Elbanna, A.M. (2016) Solid Particles Injection in Gas Turbulent Channel Flow. Energy and Power Engineering, 8, 367-388. http://dx.doi.org/10.4236/epe.2016.812032

Received: November 22, 2016

Accepted: December 18, 2016

Published: December 21, 2016

Copyright $\odot 2016$ by authors and Scientific Research Publishing Inc. This work is licensed under the Creative Commons Attribution International License (CC BY 4.0).

http://creativecommons.org/licenses/by/4.0/

\begin{abstract}
This paper represents a review of the recent researches that investigate the behavior of the gas turbulent flow laden with solid particles. The significant parameters that influence the interactions between the both phases, such as particle size, loading ratio and the gas velocity, have been extensively reviewed. Those parameters are presented in dimensionless numbers in which the applicability of studying its effect in terms of all circumstances of the gas turbulent channel flow at different condition is possible. The represented results show that the turbulence degree is proportional to the particle size. It was found that at the most flow conditions even at low mass ratio, the particle shape, density and size significantly alter the turbulence characteristics. However, the results demonstrate that the particle Reynolds number is a vital sign: the turbulence field becomes weaker if particle Reynolds number is lower than the critical limit and vies verse. The gas velocity has a strong effect on the particles settling along the channel flow and as a result, the pressure drop will be affected.
\end{abstract}

\section{Keywords}

Two-Phase Flow, Particle-Laden Flow, Gas-Solid Channel Flow, Particle Size Effect

\section{Introduction}

The multiphase flow associated with turbulence is involved into many industrial applications, such as pneumatic conveying [1] and transportation of the pulverized fuel particles in a pipeline [2] [3]. The interactions between the turbulent flow and the chaotically moving particles generate a flow of complex pattern [4] [5]. These interactions are not completely clear. The mutual interactions be- 
tween the phases in gas-solid are influential as they affect the performance of the industrial applications that involve multiphase flow. Many researchers have investigated these interactions experimentally [6] by mean of CFD codes [7] [8]. Generally multiphase flow is classified into two categories: first is the dispersed multiphase flow in which the main phase carries the dispersed phase that is distributed among the carrier phase [9], such as particles suspended in the air stream. The second is the separated flow where the phases are separated but in contact at a thin line without any mixing process during the flow, such as the annular flow in which a gaseous core flow is separated from the pipe wall by a liquid layer [10]. Multiphase modelling for fluid/particles interaction might be classified according to the degree of the phase coupling [11]. If one of the phases has an effect on the other phase but not vice versa, this flow is called one-way coupling [10]. In the two-way coupling, both of the two phases exchange the effects with each other [12]. In the four-way coupling, the effect of particles collisions is incorporated [13] [14]. The particles motion might be classified according to the mechanisms which govern the particles motion. In the first category which is the dilute phase in, the particles are suspended and their motion is governed by the fluid dynamics forces [10] [15]. The second category is the dense phase and the collision between the particles is the dominant factor [16].

The response of the particle motion to the carrier phase motion disturbance is expressed by different dimensionless parameters. The first dimensionless relation is defined as the ratio between the response time of the particle momentum change and the time apart from the particles collisions [10]. For the dilute phase, this ratio is less than unity and means that there is a sufficient time available for the particles to respond to the carrier phase dynamic forces. The second effective dimensionless number which is known as the particle Reynolds number $R_{e p}$, is defined as representing in Equation (1):

$$
R_{e p}=\left(u_{f}-u_{p}\right) d p / v
$$

in which, this number represents the ratio of fluid inertia to fluid viscosity near the particle's surface [4]. Where $u_{f}$ and $u_{p}$ indicate to the fluid and the particle velocity, respectively. The higher particle Reynolds number is, the higher turbulent intensity will be [17]. The degree of phase coupling is proportional to the particle Reynolds number. Increasing the particles Reynolds number develops the wakes behind the particles and causes that the carrier phase turbulence will be affected [10].

Similar behavior is observed as the mass loading ratio increases and the particles concentration along the pipe cross section will be increased [10] [11]. The flow patterns are affected by solid loading ratio and air mass flow rate as illustrated in Figure 1. The solid loading ratio is the ratio of dispersed phase mass flux to that of the continuous phase. As can be seen from Figure 1, the curves A, $\mathrm{B}$, and $\mathrm{C}$ represent the zero, intermediate and highest mass loading ratio, respectively. During the reduction of the air mass flow rate at a fixed loading, the phase 


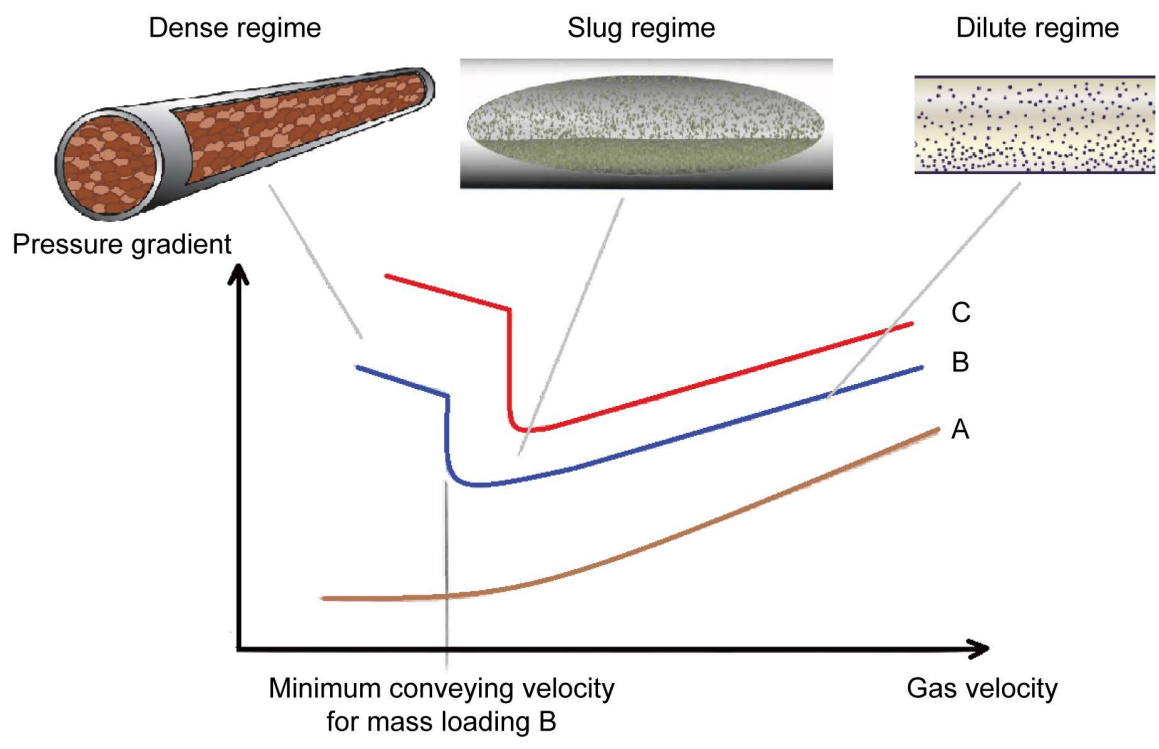

Figure 1. Flow modes diagram [15].

experiences three stages [15]. First, in the dilute phase, a reduction in the pressure is observed due to the settling of the particles in the lower half of the pipe line [18]. The settling is a consequence of the dominant gravity effect and particles inertia that is leading to the particles separation [19]. The pressure reduction continues until it reaches a minimum pressure point which is corresponding to saltation velocity of the carrier phase. The saltation velocity is the point where the drag and lift forces are unable to keep the particles suspended [10] [20]. Second, the settled particles are transported in layers. Then the transition from the dilute phase to unsteady slug phase takes place [21]. In this stage, the settled particles increase and form a stationary layer (plugs) that is transported along the lower section of the pipeline and produce a high-pressure gradient [18]. The final stage is the steady dense phase where the particles move in a gentle and quiet pattern. Operating at higher loading ratio generates a substantial increasing in pressure drop as a result of the additional resistance which is offered by the high concentrated particles [22]. The additional pressure drop leads to raising the base speed required to transport to particles. The dilute loading where the carrier phase flow controls the particles motion and the inter-particles interaction can be ignored [19] as well as the particles-wall interaction [12]. At the same time, the particles strongly affect the carrier phase flow even with low solid volume fraction [12]. The momentum exchange between the two phases takes place [11]. Therefore, in many cases, it might be appropriate to regard the dilute phase as two-way coupled flow. The mutual interaction associated with the particles and the carrier phases represents the cornerstone in predicting the performance of the multiphase industrial applications.

The aim of the present work is regarding the dilute phase turbulent flow. The dominant mechanisms which govern the particles dispersion such as, gravita- 
tional settling, drag force and the lift force, will be reviewed. Then the turbulence modulation mechanisms such as kinetic energy exchange, the dissipation rate and the secondary motion induced by the particles, will be reviewed. The two-way coupling flow, in which the turbulence affects the particles motion and vice versa, many parameters such as, particle size, particle shape and mass loading ratio, will influence the particles and the carrier phase interaction. In the context of seeking, to identify the interactions between phases and the dominant parameters, some dimensionless numbers will be illustrated. In the present work, the important results will be presented and divided into two main sections. First one is concerned with the particles dispersion and the second section will describe the turbulent modulation. We believe that, the physical reasoning of the results is essential to achieve a development in the modelling and further analysis for multiphase flow applications.

\section{Particles Dispersion}

The effect of the flow turbulence on the particles motion is called turbulent dispersion. The degree of mixing between the phases, the particles behavior and the cross-sectional distribution are strongly related to the particles dispersion effect [10]. The particles dispersion significantly affected by the particles-gas time ratio which represented by the Stokes number [10] [11]. Stokes number is an indication of the response degree of the particle to the motion alterations produced by the carrier phase turbulence [4]. The dimensionless stokes number can be defined by Equation (2).

$$
S t=\tau p / \tau g
$$

where $\tau p$ is known as the required time for the particle to accelerate from rest to $63 \%$ of the carrier flow velocity [4] and $\tau g$ is the carrier phase characteristic time scale. When $S t \ll 1$, the response time of the particles is less than carrier phase time scale, by another word the particle has enough time to respond to the velocity of the carrier phase, as a result the particles just follow the carrier phase velocity field [10]. This phenomenon is known as the characteristic of dilute mode [15], which associated with sufficient particles momentum to avoid the settlement [16]. If $S t \gg 1$, the particle does not have enough time to respond to the carrier phase velocity change [10]. However, the particle will experience a late response to the velocity changes due to the higher inertial effect of the particle [23]. As a result, the particle tends to separate from the flow [4]. In this case, the particles are subjected to inter-particles collision even at low volume fractions [11]. When $S t \approx 1$, the particles inertia is a vital parameter as well as the particles-turbulent interactions [6].

Whatever, during the transportation of the particles by mean of turbulence or drag force, the particles exhibit changes, such as, settlement and re-suspension, along with the flow that might be clearly predicted using cross-sectional distribution [15]. The settling effects interfere with the transportation of the particles 
and they will be presented separately in order to illustrate incomprehensible manner the effective parameters.

\subsection{Transport of the Particles}

The particles behavior during the transportation of the glass beads with a diameter less than $110 \mu \mathrm{m}$ through a long glass horizontal pipe with the inner diameter of $75 \mathrm{~mm}$ has been presented. The air velocity was $12 \mathrm{~m} / \mathrm{s}$ with the solid loading of $1.6 \%$ [23]. The obtained results show that the air axial velocity profile was annular with the maximum air velocity of $12.5 \mathrm{~m} / \mathrm{s}$ at the pipe center which is constant at various sections along the pipe. The obtained results illustrated in Figure 2 and show that the particles axial velocity exhibit an annular behavior with maximum speed that increases from $13.5 \mathrm{~m} / \mathrm{s}$ at $\mathrm{x}=300 \mathrm{~mm}$ to $15 \mathrm{~m} / \mathrm{s}$ at $\mathrm{x}$ $=200 \mathrm{~mm}$. A probable reason is that the large particles have been settled by gravitational settling effect, but the smaller particles still suspended within the fastest region. Since the smaller particles with lower inertial effect, however, they still seek to accelerate along the flow. As appeared in Figure 2, the settled large particles in the base of the pipe don't show a speed variety along the $\mathrm{x}$ direction. This is because of that, the large particles less sensitive the aerodynamic effects of the carrier flow and their motion dominantly driven by the collisions between them and collisions with the walls.

Another experiment was done by three glass beads with the diameter of 0 - 50 $\mu \mathrm{m}, 0-110 \mu \mathrm{m}$ and $180-300 \mu \mathrm{m}$ at the section of $\mathrm{x}=300 \mathrm{~mm}$ along the pipe flow [23]. The first diameter range shows an ability to follow the carrier phase with an axial velocity at the pipe center that is similar to the carrier phase maximum velocity. Increasing the particles size raises the difference between the particles axial velocity and the air velocity. The third diameter range in which is associated with large $S t$ Number, the particles retain their initial velocity. As a consequence, the maximum velocity of the particles at the pipe center is higher than the local air velocity. Exhibit a greater velocity difference in between the particles and the carrier phase than the other two types.
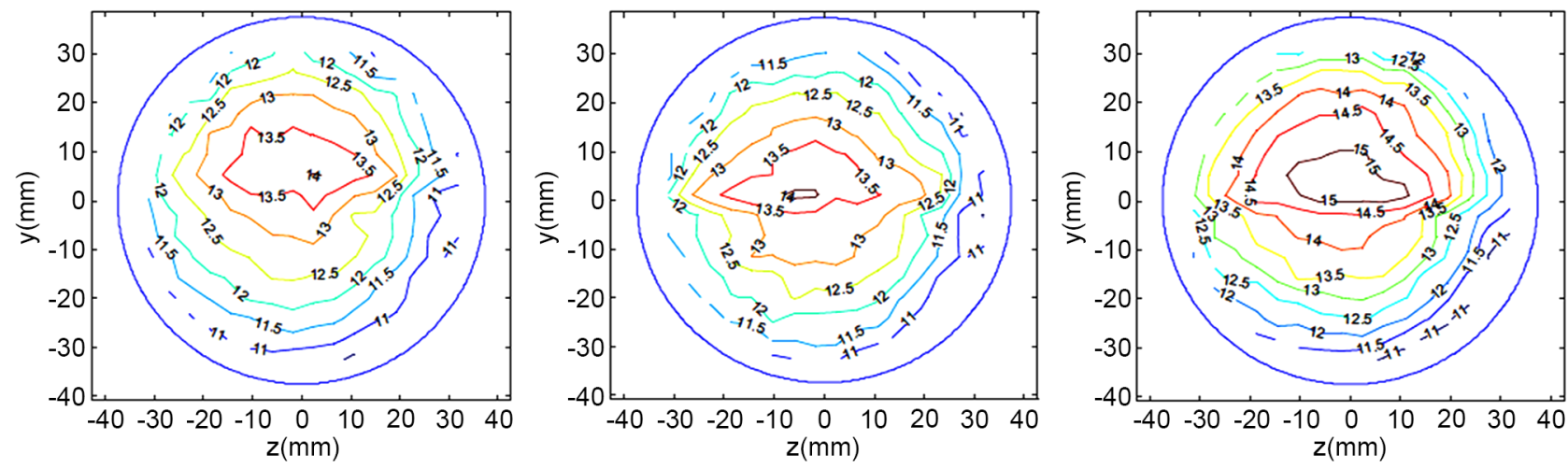

Figure 2. Contours of the axial particle velocity at sections $x=300 \mathrm{~mm}$ (left), $x=250 \mathrm{~mm}$ (middle) and $\mathrm{x}=200 \mathrm{~mm}$ (right), $\mathrm{x}$ is decreasing in the stream wise direction [23]. 
In a simulation process of horizontal flow in a pipe with the inner diameter of $30.5 \mathrm{~mm}$ [13], the results were observed at a section corresponding to a fully developed pattern. The dispersed phase was plastic pellets with the density of 1020 $\mathrm{kg} / \mathrm{m}^{3}$ and two different diameters of $200 \mu \mathrm{m}$ and $50 \mu \mathrm{m}$. With the gas velocity of $10 \mathrm{~m} / \mathrm{s}$ and mass loading ratio that is $\eta_{o}=2.2 \%$. The particles are injected with the mean velocity that is the same as the gas mean velocity. The following has been observed, the maximum mean axial velocity of the large particles is located at the pipe center. This is a consequence of the homogeneous distribution of the particles across the pipe cross section. In contrast, for smaller particles, the beak of the mean axial velocity of both phases is displaced little above the pipe center. The explanation is that the particles-wall collision frequency at the upper surface of the pipe is smaller than that at the pipe lower surface. This is a result of the high particles concentration near the bottom surface of the pipe.

The drag force has been studied experimentally with pulverized coal particles with the diameter of $125-150 \mu \mathrm{m}$ [24]. A horizontal dilute flow along a pipe with $16 \mathrm{~mm}$ inner diameter and the results were observed at a section that is far from the pipe entrance by $4.3 \mathrm{~m}$. The results show the difference between particles velocities across the pipe cross section. The particles that occupy the upper half of the pipe are faster than that occupy the lower half at a given superficial gas velocity and a solid feeding rate. The velocity difference, between the upper half and lower half particles, decreases with increasing the gas velocity. The differences become almost constant when the gas velocity is being greater than 11 $\mathrm{m} / \mathrm{s}$, due to the uniformity of the particles distribution associated with increasing the gas velocity. For constant gas velocity, as the solid feeding rate increases, the velocity differences increase. As the carrier phase would not be able to re-suspend most of the particles that are settled by the gravity action.

The flow along a horizontal rectangular cross section channel at Reynolds number of 6826 corresponding to the gas velocity of $6.603 \mathrm{~m} / \mathrm{s}$ has been examined experimentally [25]. The cross section dimensions are $3 \mathrm{~cm}$ (height) $\times 30$ $\mathrm{cm}$ (width) and $600 \mathrm{~mm}$ (length). The flow is laden by two groups of polythene beads. The first group is characterized by a diameter of $60 \mu \mathrm{m}$ with particle Reynolds number of 1.5. The second group has characterized a diameter of 110 $\mu \mathrm{m}$ with particle Reynolds number of 5.5. The particle density around 1030 $\mathrm{kg} / \mathrm{m}^{3}$ with mass loading ratio varied from $0.05 \%$ to $4 \%$. Therefore, the distance between particles is large enough to weaken the inter-particles collision effect. The low level of particles Reynolds number makes the effect of the vortex shading on turbulence is insignificant. As shown in Figure 3, the lag between the carrier phase and the particles is maximum in the core region of the flow and decreases near to the wall. The particles axial velocity decreases with increasing the mass loading. The axial particles fluctuations are always greater than the carrier phase axial fluctuations. The high gradient of the axial particles fluctuations in the vicinity of the wall is considerable. Might be explained as, particles-wall 

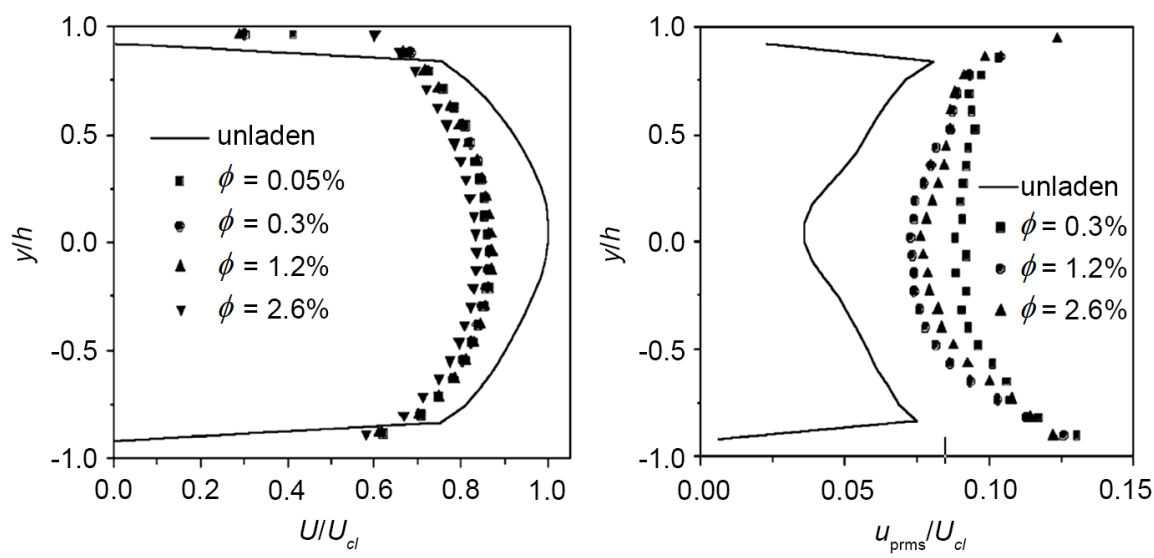

Figure 3. Particles mean axial velocity (left figure) and particles axial fluctuation velocity (right figure), particles diameter $=110 \mu \mathrm{m}$ [25].

collisions, which are lead to the radial motion of the particles, alter the axial particles fluctuations. For the larger particles, the variation is disordered, as the mass loading increases the fluctuations decrease and then increase at the highest mass loading. However, for small particles, the fluctuations are almost unchanged as the mass loading is varied.

The radial particles fluctuations level directly proportional to the solid mass loading and the particles size [25]. Confirm that, the small particles tend to keep track of the carrier phase motion under the turbulence effect. In contrast to the large particles, those are in which the inertial effect prevails the particles motion. As the particles are transported horizontally they experience a gravitational force, as a result, they tend to settle. After the collision with the bottom surface the large particles, which contain enough inertial momentum, will return bake to the main flow again. The large particles fluctuations are stronger than those of the carrier phase. While the smaller particles, those contain small momentum, will not travel in the transverse direction and retained near the walls. As a result, the transversal particles fluctuations are weaker than those of the carrier phase.

The particles shape effect is examined at lower mass loading ratios using the horizontal jet that is laden with rod-shaped nylon fibers with dimensions that are the length of $320 \mu \mathrm{m}$ and diameter of $24 \mu \mathrm{m}$ [6]. The dispersed phase density is $1.13-1.15 \mathrm{~g} / \mathrm{cm}^{3}$. The mass loading ratios were $0.002 \%$ and $0.006 \%$. At Reynolds number of 9000 . The jet extends from a circular pipe with the diameter (D) $2.2 \mathrm{~cm}$ under fully developed turbulent conditions. The axial variation of the mean velocity states that the average axial velocity of the carrier phase is higher than the particles. The velocity difference is attenuated as the jet grows toward the axial direction. The radial variation of the mean velocity as in Figure 4, states that at $x / D=5$ the velocity of the particles approach the carrier phase velocity for the inner region of the jet. This is due to that the particles maintain their speed for larger axial distance $(\mathrm{x})$ than the carrier fluid. As the jet grows toward the axial direction the dispersed phase velocity gets closer to the carrier 


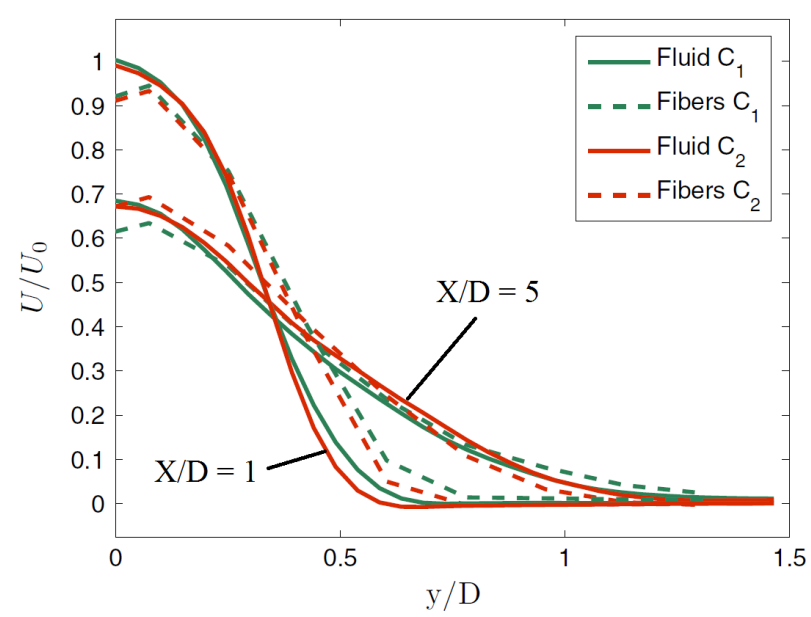

Figure 4. Radial variation of the mean axial velocity of the particles two downstream positions, $\mathrm{x} / \mathrm{D}=1$ and $\mathrm{x} / \mathrm{D}=5 ; \mathrm{C}_{1}=0.002 \%, \mathrm{C}_{2}=0.006 \%[6]$.

phase velocity. The particles exhibit a higher velocity than the carrier phase, this behavior is confined to the shear layer out of the jet core region and near to the jet boundaries where the interaction between the jet and ambient has occurred.

A parameter that is called the Particle sphericity $(\phi)$ is incorporated in a simulation study [19]. This parameter reflects how the spherical particle's surface is similar to the non-spherical particle's surface of the same volume. Non-spherical isometric quartz particles of $185 \mu \mathrm{m}$ diameter are used, with constant air velocity and low mass loading ratio. This modeling regards only the drag force is the only dominator on the particles motion. And show that, as the particles sphericity decreases, the drag coefficient increases. As a result, the particles mean velocity resembles the gas velocity. The vertical component of the particle velocity fluctuations, indicates to the collision frequency and the local momentum transfer, is proportional to the particle sphericity. When the lift effect is involved in the modeling process, the velocity of the lower half particles is increased.

Another experimental study was performed. Non-spherical quartz particles of $150 \mu \mathrm{m}$ mean diameter are injected at high Reynolds number [26]. The flow exhibits a wider range than the corresponding flow of spherical particles of the same size. The quartz particles exposed to higher drag force than the spherical particles. As a consequence, the mean and the fluctuating velocity of the quartz particles are higher and closer to the carrier phase velocity [27].

The volume fraction is defined as the ratio between the space volume occupied by the particles and the volume occupied by particles and carrier phase. The particles interactions are dominantly affected by the volume fraction. In which for spherical particles the interaction has been categorized according to the volume fractions [11]. When the volume fraction is larger than $10 \mathrm{E}-3$, the interparticles interactions have a dominant effect which known as four-way coupling. And four-way coupling is the consequence. 
The degree of the coupling that is regarded in the modeling process has its influence on the flow configuration and velocity of the particles. A numerical simulation based on both the two-way and four-way coupling is performed [13]. In the two-way modeling, the particles local mean velocity is higher than the corresponding in the four-way coupling. This effect results from regarding the effect of the in-between particles collision.

\subsection{The Particles Settling}

The gravitational settling influences the particles to cross-sectional concentration. The mean axial velocity of both phases will be influenced by the gravitational settling. In the pre-mentioned investigation [23], the particles number concentration has been investigated. As a result of the gravity, the particles number increases in the negative $y$ direction. The settling rate increases in the streetwise direction from 1400 at $\mathrm{x}=300 \mathrm{~mm}$ to 1600 at $\mathrm{x}=200 \mathrm{~mm}$ as can be shown in Figure 5. The particles injection condition has its effect on the settling. As the particles are injected from the right side of the pipe (positive z-direction) and travel across the pipe cross section, they retain some of their initial momenta. Due to the inertial behavior of the particles they are settling in the opposite direction (negative $\mathrm{z}$-axis).

The particles size effect on the settling is shown in Figure 6. Since the larger size particles inertia is higher, their motion is almost completely under the control of collision effect while the turbulence weakly affects the particles [13]. As a result, the particles are homogeneously diffused through the cross section. In contrast, the smaller particles are dominantly influenced by the gas turbulence and the gravitational settling. As a result, they concentrate in the lower section of the pipe.

Another result was observed, as the mass loading ratio increases, the particles concentration near to the bottom surface of the pipe decreases. This effect is a consequence of the enhancement of the inter-particles collisions rate.
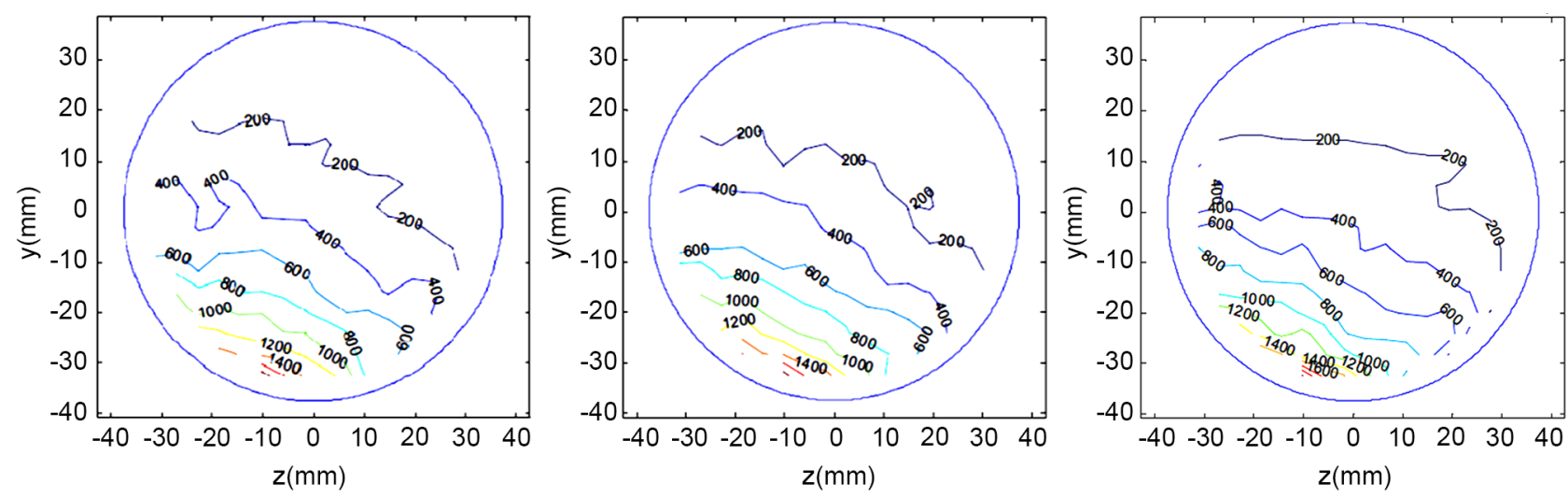

Figure 5. Contours of particle number rate at sections $x=300 \mathrm{~mm}$ (left), $x=250 \mathrm{~mm}$ (middle) and $\mathrm{x}=200 \mathrm{~mm}$ (right), $\mathrm{x}$ is decreasing in the stream wise direction [23]. 
Experimental work was performed to illustrate the air pressure drop along the flow that is generated by the settling effect [22]. Horizontal flow through a pipe with a diameter of $117 \mathrm{~mm}$ and length of $2.7 \mathrm{~m}$ has been used. The flow is laden by Polystyrene particles of cylindrical shape with $3.2 \mathrm{~mm}$ diameter and density of $1.050 \mathrm{~kg} / \mathrm{m}^{3}$. The mass loading ratio is varied from 0.06 to $0.11 \mathrm{~kg}$ particles $/ \mathrm{kg}$ gas. And the solid mass flow rate varied from 0.085 to $0.17 \mathrm{~kg} / \mathrm{s}$. The air velocity ranges from 5 to $35 \mathrm{~m} / \mathrm{s}$. The higher mass loading the higher pressure drop and higher air speed corresponding to minimum pressure drop as illustrated in Figure 7. This effect is a result of that, the high resistance offered by the high concentration particles that are associated with the higher mass loading. Another appropriate explanation is, increasing the mass loading leads up to the higher rate of particles-wall collisions and consequently, the particle momentum got lost [28]. At a fixed loading ratio, the pressure drop is directly proportional to the carrier phase velocity due to the frictional effect and flow disturbance. As the carrier phase, velocity decreases the pressure drop decreases until reaches the lowest pressure drop. A reflexion point is observed where the pressure drop
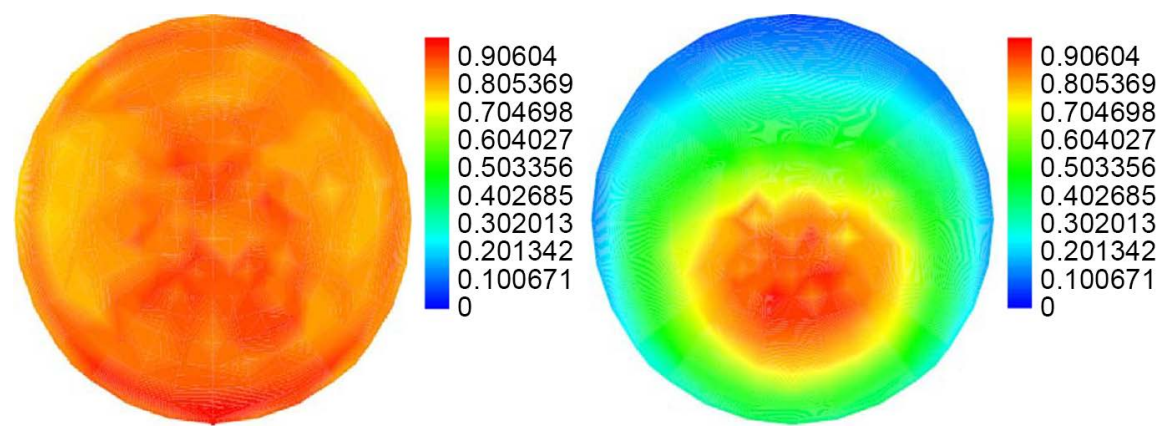

Figure 6. Particles mass concentration, with particles diameter of $D_{p}=200 \mu \mathrm{m}$ (left figure) and $D_{p}=50 \mu \mathrm{m}$ (right figure) and mass loading ratio $\eta_{o}=2.2$. Four-way coupling [13].

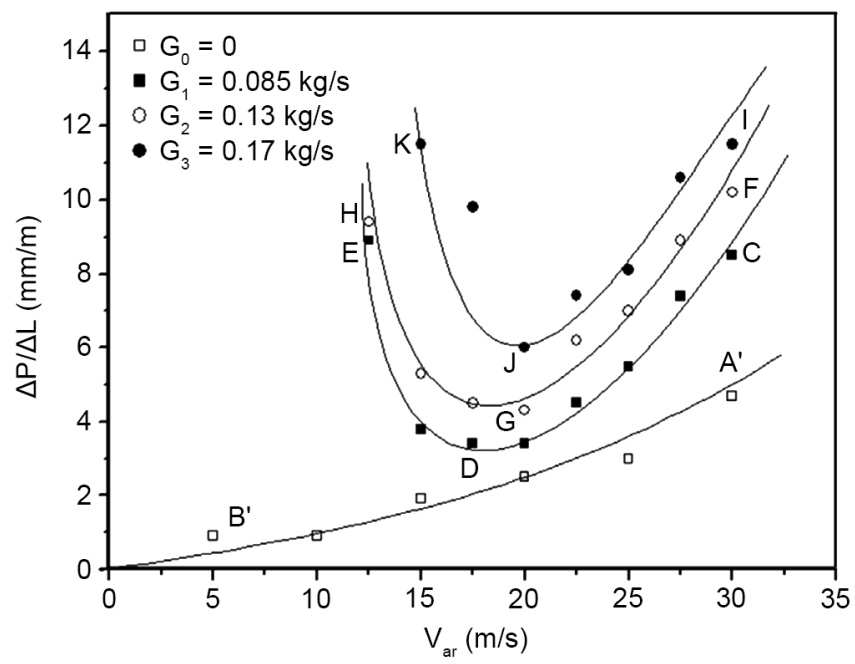

Figure 7. Air pressure drop variation with the air velocity and the mass loading [22]. 
starts to increase again as a result of the particles settling in the vicinity of the lower surface of the pipe. The particles settlement is a consequence of that, as the air velocity is reduced its ability to suspend the particles is consequently reduced.

The results of another study show the pressure drop along a horizontal flow of $4 \mathrm{~mm}$ particles with air velocity ranges from 15 to $30 \mathrm{~m} / \mathrm{s}$ and loading ratio ranges from 0.5 to 2.5 [28]. A similar curve of the pressure drop was observed. Explained as, the collision of the particles creates a difference between the air and the particles axial velocity consequently the particles exhibit a higher drag force. Although the operating conditions extend to low mass loading and high air velocity, considerable particles concentration in the lower half of the pipe [22].

\section{Turbulent Modulation}

The presence of the discrete phase alters the carrier phase turbulence; this effect is called the turbulent modulation. Such as that the mean velocity of the carrier phase is affected by the particles hence the turbulence production rate and the mean strain rate will be changed [10]. As the mean, relative velocity between the particles and the carrier phases increases, the energy begins to transfer to the smaller scales velocity fluctuations of the continuum main flow stream. The turbulence modulation is associated with many mechanisms, such as turbulent kinetic energy transfer, turbulent dissipation rate (turbulence attenuation by particles) and the secondary motion induced by the particles. Such mechanisms are dependent on parameters such as particle size and shape, mass loading and the carrier phase velocity.

\subsection{The Inertia Effect}

The classification of the interaction between the phases is examined [11]. As shown in Figure 8, when the volume fraction $(\phi)$ is located between $10 \mathrm{E}-6$ and $10 \mathrm{E}-3$, the consequence is the two-way coupling. Whereas that the turbulence structure is strongly influenced by the particles due to the momentum exchange between the particles and the carrier phase. The particles inertial effect might be appropriately expressed using a dimensionless number that represents the particle response time $\tau p$ to Kolmogorov time scale $\tau k$. For the two-way coupling range represented by zone A, which is characterized by $R_{e p} \leq 1$ and $\tau p / \tau k \leq 10$, the particles influence on the turbulence is dependent on $\tau p / \tau k$. For the microparticles that are characterized by $\tau p / \tau k \leq 0.1$ the turbulent kinetic energy and the dissipation rate are greater relative to the corresponding in the single phase flow. The ghost particles where $0.1 \leq \tau p / \tau k \leq 0.5$ the turbulent kinetic energy is still as it is without change but the dissipation rate is greater than the corresponding in the single phase flow. The critical particles where $\tau p / \tau k \approx 1$, the turbulent kinetic energy is reduced but the dissipation rate is still as it is without change. The large particles with $\tau p / \tau k \geq 1$ the turbulent kinetic energy 


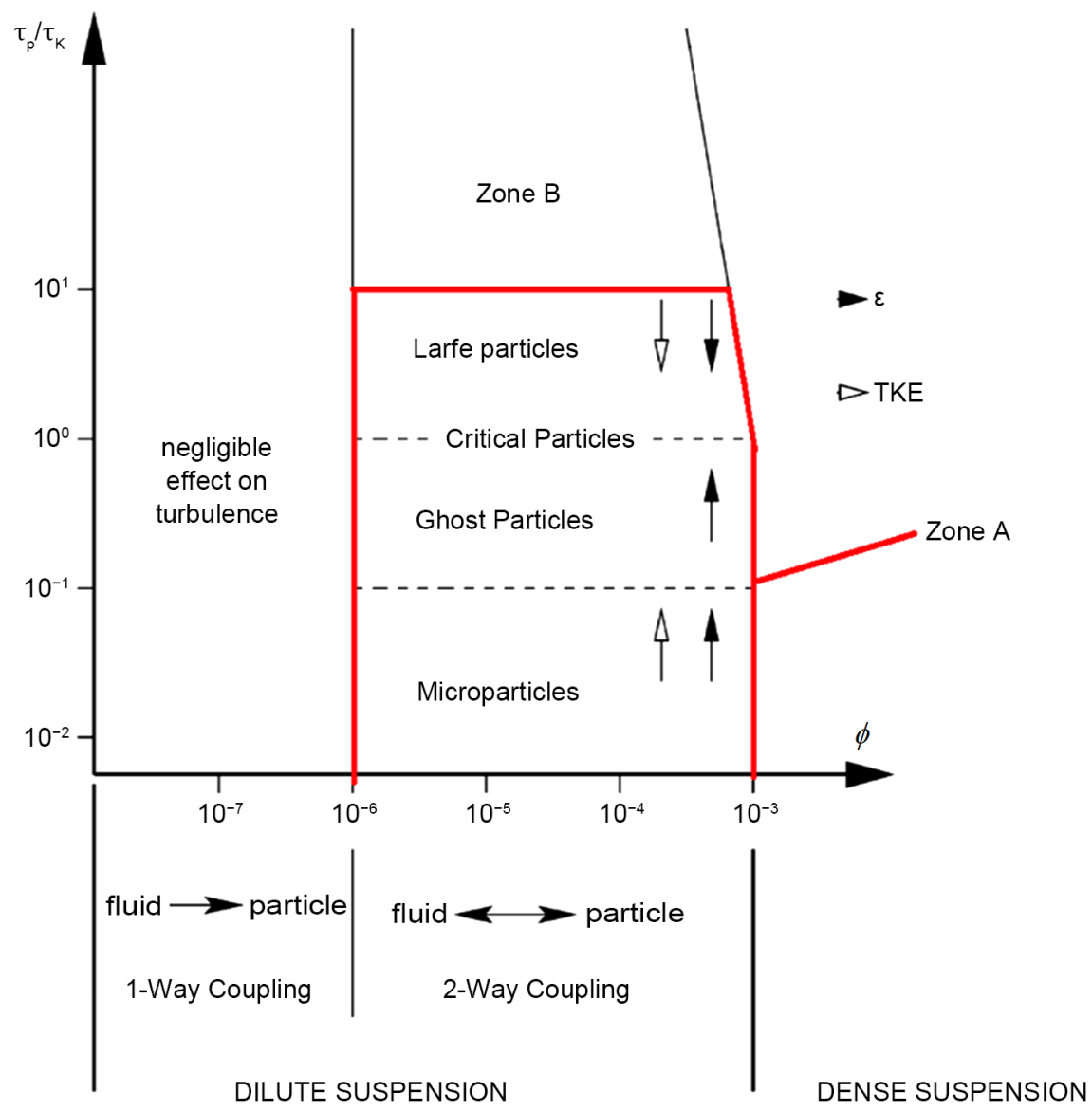

Figure 8. Classification map of particle-laden turbulent flows [11].

and the dissipation rate are reduced relative to the corresponding in the single phase flow. For zone $\mathrm{B}$ that is characterized by higher $R_{e p}$ associated with the further increase of $\tau p$ by increasing the particles size.

At $R_{e p} \geq 400$, vortex shading appears. These wakes which are generated behind the particles begin to grow at high particle Reynolds number [10]. Lead to transform some of the particles kinetic energy into turbulence [4]. Thus, enhance the turbulent energy production. The particle Reynolds number is might be used to express the carrier phase inertia effect around the particles. The turbulence field of the carrier phase is reduced if the particle Reynolds number is low and vice versa [29]. A study that is used spherical particles as dispersed phase and shows that. For $R_{e p}<200$, the turbulent intensity exhibits reduction whereas for $R_{e p}>400$ the turbulent intensity increases [30].

The appearance of the vortex that is induced by the particles observed at $R_{e p} \approx$ 270. Another investigation shows that for spherical particles the vortex shading appears at $R_{e p}>400$ [31].

A characteristic length ratio has been used to express the turbulent modulation [32]. This ratio defined as the ratio between the particle diameter and the length scale of the eddy. And found that when this ratio is greater than 0.1 the 
turbulence intensity increased while when it is lower than 0.1 the turbulence is decreased.

As mentioned previously, the larger particles are subjected to the higher inertial effect [23]. Thus, they are not perfect tracker to the eddies motion of smaller scales [33].

For turbulent jet flow laden by particles of different sizes [7], the distance that extends from the orifice to the point that is corresponding to the velocity of the maximum particles represents the particles developing the region. In this region, the TKE continues in decreasing and the velocity of the particles increases, due to the high drag force that exerted by the gas on the particles. After the particles developing region the TKE increases, in a relatively steep manner, as well as the flow velocity decreases. This is a consequence of that, the surrounding air sweeps along in the jet center. Finally, the TKE is decreased in a relatively steep manner. The large particles enhance the carrier phase turbulence where the small particles reduce the turbulence [34]. An explanation is that the large particles will transfer its stored potential and kinetic energy to the adjacent fluid. Another supporter reason is that, the vortex that is induced by the large particles. In the other hand, the large particles need the greater amount of energy to be forced to move along the flow direction [4]. The small particles forced to go after the turbulent eddies under the effect of the drag force. As a result, the small particles extract some energy from eddies. While the wakes, which are generated by the small particles, are not large enough to compensate the extracted energy and the turbulence is attenuated.

The carrier phase exhibits a higher mean axial velocity when it is laden by particles [6]. This observed clearly at the axial distance from the jet outlet that is $\mathrm{x} / \mathrm{D}>2$ where $\mathrm{D}$ is the outlet diameter. Radial variation of the mean axial velocity of the carrier phase shows that; at $x / D=1$ the laden velocity is higher than unleaded; this difference is confined in the shear layer. While for $x / D=5$ the difference is maximized at the jet axis and is decreased toward the radial direction.

At the jet outlet, the following were observed; the mean velocity is decreasing along the streamwise while the turbulence is developing [6]. The axial fluctuations velocity level of the carrier phases is enhanced in the laden case as compared with the unladed case. The maximum increasing of the turbulence intensity occurs at the jet core.

Gas jet turbulent flow laden with solid was studied using direct numerical simulation with high Reynolds number [35]. In the case of particles with stokes number of 0.01 and 50 the appearance of large scale vortex is advanced and the level of the turbulence intensity becomes lower. However, for stokes number equals to unity the appearance of large scale vortex is delayed.

The turbulent modification is strongly influenced by the particles concentration within the cross section [25]. The non-uniform particles concentration is 
observed, especially for the large particles due to the dominant inertial effect. The mean axial velocity of the carrier phase is decreased as the particle size increases. The carrier phase axial velocity near the wall is greater in the case of the laden flow than the unloaded flow. This is a consequence of the drag force applied by the particles on the flow at the wall region. The velocity gradient of the carrier phases in the vicinity of the walls is greater in the case of laded flow. This is an appropriate condition to generate the turbulence. The carrier phase axial fluctuation velocity is enhanced when the flow is laded. For the small particles, the turbulence intensity variation is completely influenced by the interactions between phases. In the case of $60 \mu \mathrm{m}$ particles, the intensities decrease in a steep manner at the wall region.Then the intensities attenuating rate is decreasing until it reaches to the point of inversion located at the center line. In the case, if 110 $\mu \mathrm{m}$ the variation is clearer and the turbulent intensities are proportional to the mass loading.

The particles size effect has been examined by using the horizontal flow of plastic particles through a $30 \mathrm{~mm}$ pipe [36]. The particles with diameters of 3.4 $\mathrm{mm}$ and $0.2 \mathrm{~mm}$ at $R_{e p}=470$. As shown in Figure 9, the large particles with a diameter of $3.4 \mathrm{~mm}$ enhance the turbulence intensities of the carrier phase. The asymmetrical radial variation of the turbulence intensity confirms that, the particles distribution is not symmetrical. This is expected since the gravitational force influences the particles concentration. While the turbulence intensities are reduced in the case of small particles of $0.2 \mathrm{~mm}$.

At $R_{e p}=25$ the effect of particles size is investigated by the downward jet of air that laden with particles of different sizes [37]. The axial velocity fluctuations of air are plotted as a radial distribution at $\mathrm{x} / \mathrm{D}=20$ as in Figure 10. The turbulence level that is corresponding to the large particles of $850-1200 \mu \mathrm{m}(\mathrm{A})$ is higher than that of small particles of $180-250 \mu \mathrm{m}(\mathrm{E})$.

The axial variation of the turbulence intensity at the jet centreline has been plotted in Figure 11 [38]. The case of small particles the intensity level has been
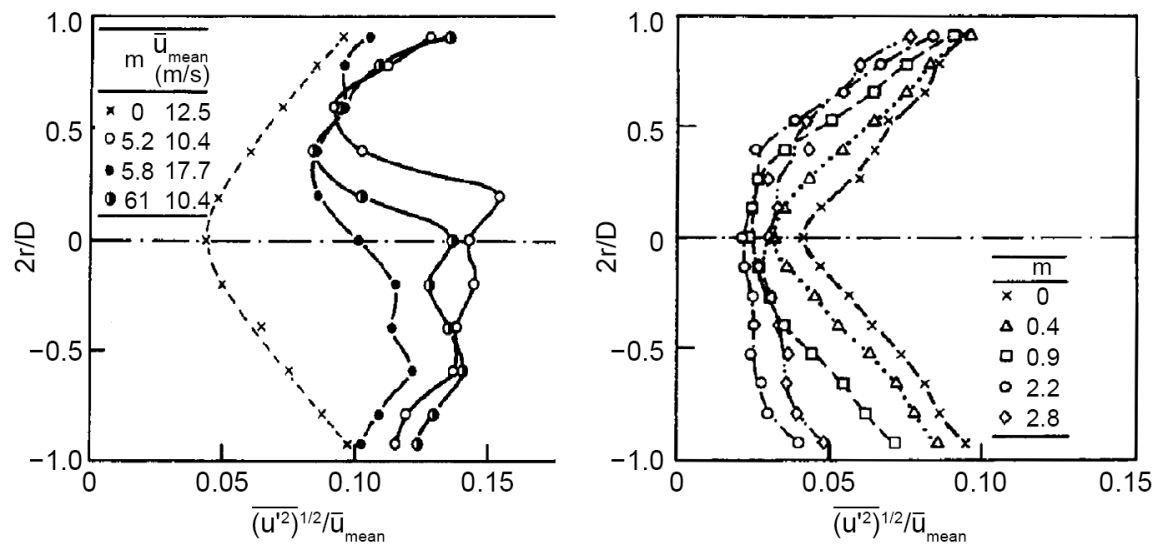

Figure 9. Carrier phase turbulence intensity, $3.4 \mathrm{~mm}$ (left figure); $0.2 \mathrm{~mm}$ (right figure) particles [36]. 


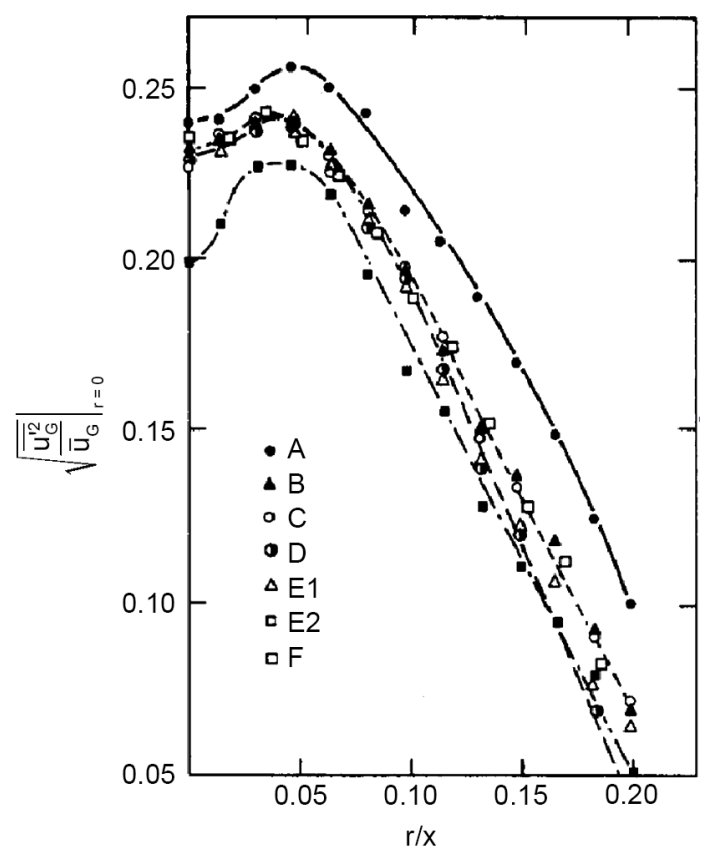

Figure 10. Radial variation of air turbulence intensity in an axial jet laden with particles of different sizes [37].

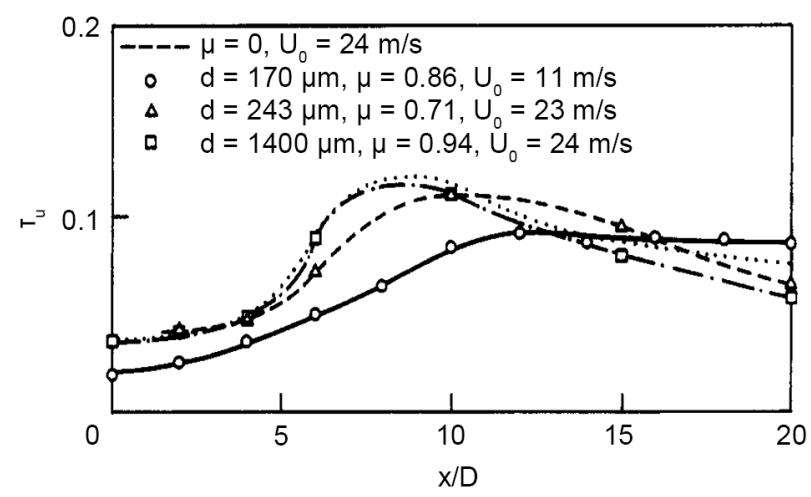

Figure 11. Axial variation of the air turbulence intensity at the jet centreline [38].

decreased. For the large particles, the turbulence intensity is enhanced. This is observed clearly as $\mathrm{x} / \mathrm{D}$ varies from 5 to 10 .

The secondary motion induced by the unbalanced forces which are induced by the particles that are suspended in the core of dilute horizontal flow. The strength of secondary motion, which in form of circulation cells, is proportional to the pipe wall roughness [13]. The influence of the wall roughness appears as the re-suspension of the particles which tend to settle in the lower section of the pipe. However, this effect is associated with increasing the collision frequency [19]. As a result of the local high momentum transfers to the gas at the bottom of pipe cross section. The pre-mentioned simulation process that is performed for the fully developed flow of gas with the velocity of $10 \mathrm{~m} / \mathrm{s}$ and mass loading ratio of $\eta_{o}=2.2$. With the retention of the same initial velocity condition for 
both air and particles, as shown in Figure 12, four recirculation cells are formed in the case large particles, as consequence of the dominant collision effect. And the gas maximum axial velocity is located at pipe center due to the homogeneous distribution of the particles. In the case of the small particles, the gas maximum axial velocity is displaced little above the pipe center. Since, as mentioned previously, the small particles concentrate in the lower section of the pipe. The higher solid mass concentration in the bottom is augmenting the local inter-particles and particles-wall collision frequency. As a result, high local momentum transfer to the carrier phase has occurred at the bottom of the pipe. While a weak probability of particle collisions at the top section of the pipe. As a result, two recirculation cells are formed near to the bottom surface of the pipe.

As the particle mass increases, with the large value of $S t$, the particles are lagging to respond to the carrier phase fluctuations. As a result, there is a relative motion is generated between the carrier phase and the particles that are dominantly generated by the fluctuations of the carrier phase [11]. The particles have a large inertia that forces the particle to resist the carrier phase fluctuations; as a result, some of the turbulence of the carrier phase is transferred into the particles and raise irregularly their kinetic energy.

An experimental study illustrates the effect of the particles shape on the turbulence modulation [39]. The interactions between the particles and the carrier phases are more complicated in the case of the non-spherical particles than the corresponding to the spherical particles [40]. Two shapes were used, prolate ellipsoidal and spherical particles. The observed result was that the spherical particles cause more reduction of the turbulence kinetic energy. As a consequence of that, the lower amount of energy losses associated with the non-spherical particles as well as the higher amount of turbulent kinetic energy that is transferred to the smaller scales [4]. The particles motions, which in turn affect secondary flow pattern, are dominantly influenced by the Reynolds number and the particles shape.
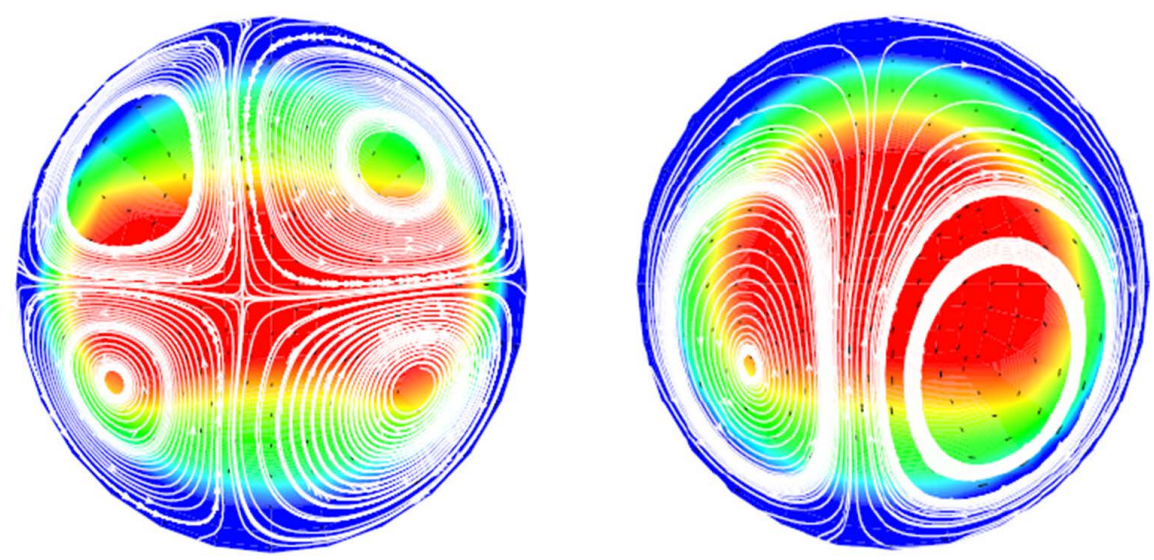

Figure 12. The secondary flow and gas mean axial velocity, with particles diameter of $D_{p}$ $=200 \mu \mathrm{m}$ (left figure) and $D_{p}=50 \mu \mathrm{m}$ (right figure). Four-way coupling [13]. 
Another experimental study was performed and found that for the spherical particles the turbulence intensity is enhanced at the core region of the pipe and while near the pipe wall the turbulence intensity is attenuated [41].

\subsection{The Particles Intensity Effect}

The mass loading is defined as the ratio between the dispersed phase mass flow rates to the corresponding of the carrier phase [10]. In the pre-mentioned study of the particle laden jet, the loading effect is incorporated [7]. For a fixed size of particles, as the solid loading increase the turbulence energy attenuates.

The turbulence is influenced by the particles even at the low mass ratio of $0.05 \%$ [25]. The mean axial velocity of the carrier phase is decreased as the mass loading increases. As shown in Figure 13, the carrier phase radial intensities are enhanced when the flow is laded [25]. In the case of large particles, the radial intensities are enhanced as the mass loading increases. However, near to the upper wall, the radial intensities decrease with increasing the mass loading. In the case of $60 \mu \mathrm{m}$ particles, the radial intensities are enhanced as the mass loading increases. However, in the case of mass loading $0.4 \%$, the intensities are closer to the unloaded flow. For the highest mass loading ratio, the turbulence intensity in the lower section of the channel is lower than that in the upper section. This effect is as result of the higher particles concentration in the lower section of the channel.

The effect of small mass loading ratio has been investigated [42]. The turbulence is decreased within the whole of the cross section of the pipe. It is Associated with a momentum transfers from the core region of the flow to the boundaries.

A result that was observed during the simulation process of the secondary motion pattern is affected by the mass ratio [13]. The inter-particles collisions rate hence the sharpness of the secondary flow is proportional to the solid mass loading ratio. As shown in Figure 14, a primary recirculation cell is generated
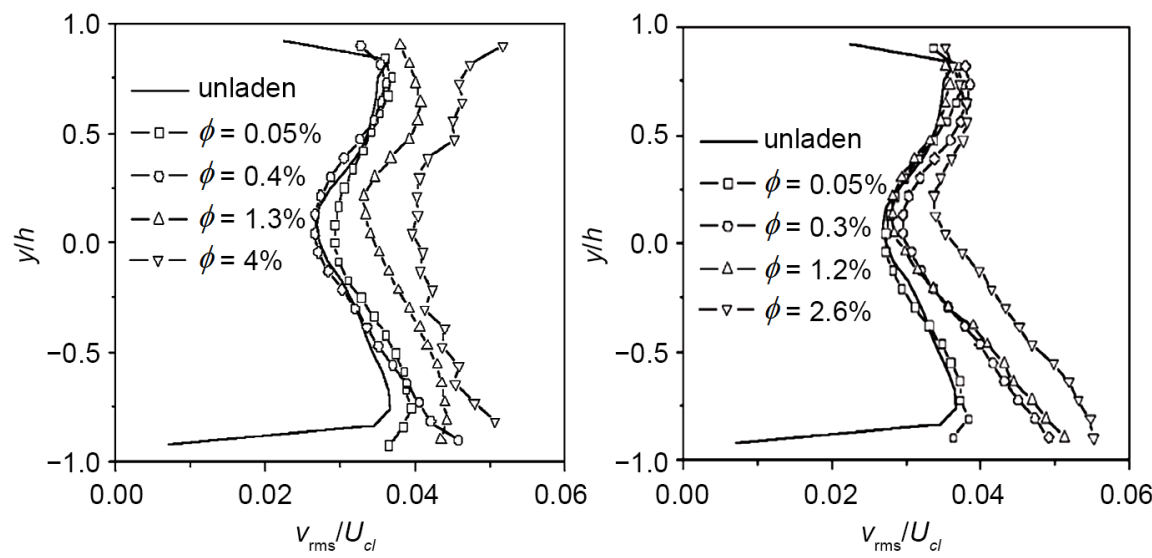

Figure 13. Carrier phase radial fluctuation velocity for particles of $60 \mu \mathrm{m}$ (left figure); 110 $\mu \mathrm{m}$ (right figure) diameter [25]. 

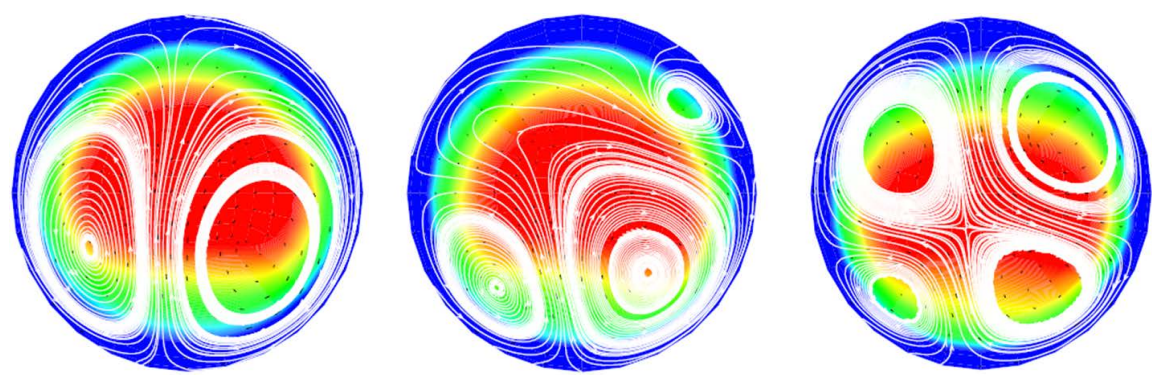

Figure 14. The secondary flow and gas mean axial velocity, with particles diameter of $D_{p}$ $=50 \mu \mathrm{m}$, at $\eta=\eta_{o}$ (left figure), $\eta=2 \eta_{o}$ (middle figure) and $\eta=4 \eta_{o}$ (right figure). Four-way coupling [13].

near to the upper half of the pipe due to the high local momentum which is transferred to the gas. However, the particles concentration near to the bottom surface of the pipe is inversely proportional to the mass loading ratio. As a result, more particles will be attended in the core of the flow. This will enhance the collision at the upper half of the pipe and high local momentum is transferred to the gas.

When the volume fraction is located between $10 \mathrm{E}-6$ and $10 \mathrm{E}-3$, the consequence is the two-way coupling [11]. However, the turbulence structure is strongly affected by the particles due to the momentum exchange between the particles and the carrier phase. For very small particles at low volume fractions, the turbulence is generated in a similar manner of single phase flow [4].

\section{Conclusions}

The aim of the paper is to review of the recent researches that investigate the behavior of the gas turbulent flow laden with solid particles. The significant parameters that influence the interactions between the both phases, such as particle size, loading ratio and the gas velocity, have been extensively reviewed. Those parameters are presented in dimensionless numbers in which the applicability of studying its effect in terms of all circumstances of the gas turbulent channel flow at different condition is possible. The investigations of multiphase flow using the CFD means to show a good validation to the experimental results, thus promise a further development for the analysis of the interactions between phases.

The presented results show that the lag between the carrier phase and the particles is maximum in the core region of the flow and decreases near to the wall. The particles size is an important parameter. Increasing the particles size raises the difference between the particles and the air velocity. The large particles are able to retain their initial velocity through the flow. Furthermore, the maximum velocity of large particles which are located at the pipe center is higher than the local air velocity. The small particles tend to follow the carrier phase motion under the turbulence effect. And the beak of the mean axial velocity of both phases is displaced little above the pipe center. The particles that occupy the up- 
per half of the pipe are faster than those occupy the lower half at a given superficial gas velocity and solid feeding rate. The velocity difference between the upper half and lower half particles, decreases with increasing the gas velocity. For constant gas velocity, as the solid mass loading increases, the velocity differences increase. As the carrier phase would not be able to re-suspend most of the particles that are settled by the gravity action. The pressure drop along the flow is proportional to the carrier phase velocity and the solid mass loading. The higher mass loading leads to that the particles concentration near to the bottom surface of the pipe decreases. The non-spherical particles are exposed to higher drag force and their velocity resembles the carrier phase velocity.

The particle Reynolds number might be used to express the carrier phase inertia effect around the particles. The turbulence field of the carrier phase is reduced if the particle Reynolds number is low and vice versa. The large particles enhance the carrier phase turbulence where the small particles reduce the turbulence. The strength of secondary flow in form of circulation cells is proportional to particles size. In the case of the large particles, four recirculation cells are formed. Two recirculation cells are formed for the small particles. The mean axial velocity of the carrier phase is decreased as the mass loading increases. Carrier phase radial fluctuation velocity is enhanced with the increasing of the mass loading as well as the secondary flow intensity.

\section{Acknowledgements}

The authors gratefully acknowledge the financial support officers from the office of the Tanta University Research Fund. This work was supported by the Tanta University Research Fund under the research grant (code: cod-tu; 03-15-02).

\section{References}

[1] Molerus, O. (1996) Overview: Pneumatic Transport of Solids. Powder Technology, 88, 309-321. https://doi.org/10.1016/S0032-5910(96)03136-1

[2] Gubba, S., Ingham, D., Larsen, K.J., Ma, L., Pourkashanian, M., Qian, X., Williams, A. and Yan, Y. (2012) Investigations of the Transportation Characteristics of Biomass Fuel Particles in a Horizontal Pipeline through CFD Modelling and Experimental Measurement. Biomass and Bioenergy, 46, 492-510.

https://doi.org/10.1016/j.biombioe.2012.07.010

[3] Chen, C.-W. (2005) Numerical Analysis for the Multi-Phase Flow of Pulverized Coal Injection inside Blast Furnace Tuyere. Applied Mathematical Modelling, 29, 871-884. https://doi.org/10.1016/j.apm.2004.11.004

[4] Saber, A., Lundström, T.S. and Hellström, J.G.I. (2015) Turbulent Modulation in Particulate Flow: A Review of Critical Variables. Engineering, 7, 597-609. https://doi.org/10.4236/eng.2015.710054

[5] Klett, J.D. (1995) Orientation Model for Particles in Turbulence. Journal of the Atmospheric Sciences, 52, 2276-2285. https://doi.org/10.1175/1520-0469(1995)052<2276:OMFPIT>2.0.CO;2

[6] Capone, A., Romano, G.P. and Soldati, A. (2015) Experimental Investigation on In- 
teractions among Fluid and Rod-Like Particles in a Turbulent Pipe Jet by Means of Particle Image Velocimetry. Experiments in Fluids, 56, 1-15. https://doi.org/10.1007/s00348-014-1876-4

[7] Patro, P. and Dash, S.K. (2014) Computations of Particle-Laden Turbulent Jet Flows Based on Eulerian Model. Journal of Fluids Engineering, 136, Article ID: 011301.

[8] Mandø, M., Lightstone, M., Rosendahl, L., Yin, C. and Sørensen, H. (2009) Turbulence Modulation in Dilute Particle-Laden Flow. International Journal of Heat and Fluid Flow, 30, 331-338. https://doi.org/10.1016/j.ijheatfluidflow.2008.12.005

[9] Balachandar, S. and Eaton, J.K. (2010) Turbulent Dispersed Multiphase Flow. Annual Review of Fluid Mechanics, 42, 111-133. https://doi.org/10.1146/annurev.fluid.010908.165243

[10] Crowe, C.T. (2005) Multiphase Flow Handbook. Vol. 59, CRC Press, Boca Raton. https://doi.org/10.1201/9781420040470

[11] Elgobashi, S. (2004) An Updated Classification Map of Particle-Laden Turbulent Flows. IUTAM Symposium on Computational Approaches to Multiphase Flow, Argonne National Laboratory, Lemont, 4-7 October 2004. https://doi.org/10.1007/1-4020-4977-3_1

[12] Eaton, J.K. (2009) Two-Way Coupled Turbulence Simulations of Gas-Particle Flows Using Point-Particle Tracking. International Journal of Multiphase Flow, 35, 792800. https://doi.org/10.1016/j.ijmultiphaseflow.2009.02.009

[13] Lain, S., Sommerfeld, M. and Quintero, B. (2009) Numerical Simulation of Secondary Flow in Pneumatic Conveying of Solid Particles in a Horizontal Circular Pipe. Brazilian Journal of Chemical Engineering, 26, 583-594. https://doi.org/10.1590/S0104-66322009000300014

[14] Lain, S., Sommerfeld, M. and Kussin, J. (2002) Experimental Studies and Modelling of Four-Way Coupling in Particle-Laden Horizontal Channel Flow. International Journal of Heat and Fluid Flow, 23, 647-656. https://doi.org/10.1016/S0142-727X(02)00160-1

[15] Fokeer, S., Kingman, S., Lowndes, I. and Reynolds, A. (2004) Characterisation of the Cross Sectional Particle Concentration Distribution in Horizontal Dilute Flow Conveying-A Review. Chemical Engineering and Processing. Process Intensification, 43, 677-691. https://doi.org/10.1016/S0255-2701(03)00096-5

[16] Hong, J., Shen, Y. and Tomita, Y. (1995) Phase Diagrams in Dense Phase Pneumatic Transport. Powder Technology, 84, 213-219. https://doi.org/10.1016/0032-5910(95)02996-F

[17] Hetsroni, G. (1989) Particles-Turbulence Interaction. International Journal of Multiphase Flow, 15, 735-746. https://doi.org/10.1016/0301-9322(89)90037-2

[18] Pan, R. (1999) Material Properties and Flow Modes in Pneumatic Conveying. Powder Technology, 104, 157-163. https://doi.org/10.1016/S0032-5910(99)00044-3

[19] Laín, S. and Sommerfeld, M. (2007) A Study of the Pneumatic Conveying of Non-Spherical Particles in a Turbulent Horizontal Channel Flow. Brazilian Journal of Chemical Engineering, 24, 535-546. https://doi.org/10.1590/S0104-66322007000400007

[20] Tashiro, H., Peng, X. and Tomita, Y. (1997) Numerical Prediction of Saltation Velocity for Gas-Solid Two-Phase Flow in a Horizontal Pipe. Powder Technology, 91, 141-146. https://doi.org/10.1016/S0032-5910(96)03250-0

[21] Tomita, Y. and Asou, H. (2009) Low-Velocity Pneumatic Conveying of Coarse Par- 
ticles in a Horizontal Pipe. Powder Technology, 196, 14-21. https://doi.org/10.1016/j.powtec.2009.06.012

[22] Santos, S., Tambourgi, E., Fernandes, F., Moraes Jr., D. and Moraes, M. (2011) Dilute-Phase Pneumatic Conveying of Polystyrene Particles: Pressure Drop Curve and Particle Distribution over the Pipe Cross-Section. Brazilian Journal of Chemical Engineering, 28, 81-88. https://doi.org/10.1590/S0104-66322011000100010

[23] Lu, Y., Glass, D.H. and Easson, W.J. (2009) An Investigation of Particle Behavior in Gas-Solid Horizontal Pipe Flow by an Extended LDA Technique. Fuel, 88, 25202531. https://doi.org/10.1016/j.fuel.2009.02.038

[24] Wang, W., Guan, Q.L., Wu, Y.X., Yang, H.R., Zhang, J.S. and Lu, J.F. (2011) Experimental Study on the Solid Velocity in Horizontal Dilute Phase Pneumatic Conveying of Fine Powders. Powder Technology, 212, 403-409.

https://doi.org/10.1016/j.powtec.2011.06.014

[25] Wu, Y., Wang, H., Liu, Z., Li, J., Zhang, L. and Zheng, C. (2006) Experimental Investigation on Turbulence Modification in a Horizontal Channel Flow at Relatively Low Mass Loading. Acta Mechanica Sinica, 22, 99-108. https://doi.org/10.1007/s10409-006-0103-9

[26] Morikita, H., Suzuki, K., Hishida, K. and Maeda, M. (1998) Dispersion of Spherical and Nonspherical Particles in a Round Jet Measured by Shadow Doppler Velocimetry. The 3 rd International Conference on Multiphase Flow (ICMF98), Lyon, 1998.

[27] Mckay, G., Murphy, W. and Hillis, M. (1988) Settling Characteristics of Discs and Cylinders. Chemical Engineering Research \& Design, 66, 107-112.

[28] Vásquez, N., Jacob, K., Cocco, R., Dhodapkar, S. and Klinzing, G.E. (2008) Visual Analysis of Particle Bouncing and Its Effect on Pressure Drop in Dilute Phase Pneumatic Conveying. Powder Technology, 179, 170-175. https://doi.org/10.1016/j.powtec.2007.06.015

[29] Geiss, S., Dreizler, A., Stojanovic, Z., Chrigui, M., Sadiki, A. and Janicka, J. (2004) Investigation of Turbulence Modification in a Non-Reactive Two-Phase Flow. EXperiments in Fluids, 36, 344-354. https://doi.org/10.1007/s00348-003-0729-3

[30] Mandø, M. (2009) Turbulence Modulation by Non-Spherical Particles. Ph.D. Thesis, Aalborg Universitet, Aalborg.

[31] Achenbach, E. (1974) Vortex Shedding from Spheres. Journal of Fluid Mechanics, 62, 209-221. https://doi.org/10.1017/S0022112074000644

[32] Gore, R. and Crowe, C.T. (1989) Effect of Particle Size on Modulating Turbulent Intensity. International Journal of Multiphase Flow, 15, 279-285. https://doi.org/10.1016/0301-9322(89)90076-1

[33] Owen, P. (1969) Pneumatic Transport. Journal of Fluid Mechanics, 39, 407-432. https://doi.org/10.1017/S0022112069002242

[34] Tanaka, T. and Eaton, J.K. (2010) Sub-Kolmogorov Resolution Partical Image Velocimetry Measurements of Particle-Laden Forced Turbulence. Journal of Fluid Mechanics, 643, 177-206. https://doi.org/10.1017/S0022112009992023

[35] Luo, K., Fan, J. and Cen, K. (2005) Modulations on Turbulent Characteristics by Dispersed Particles in Gas-Solid Jets. Proceedings of the Royal Society of London A: Mathematical, Physical and Engineering Sciences, 2005, 3279-3295. https://doi.org/10.1098/rspa.2005.1517

[36] Tsuji, Y. and Morikawa, Y. (1982) LDV Measurements of an Air-Solid Two-Phase Flow in a Horizontal Pipe. Journal of Fluid Mechanics, 120, 385-409. 
https://doi.org/10.1017/S002211208200281X

[37] Levy, Y. and Lockwood, F. (1981) Velocity Measurements in a Particle Laden Turbulent Free Jet. Combustion and Flame, 40, 333-339.

https://doi.org/10.1016/0010-2180(81)90134-6

[38] Tsuji, Y., Morikawa, Y., Tanaka, T., Karimine, K. and Nishida, S. (1988) Measurement of an Axisymmetric Jet Laden with Coarse Particles. International Journal of Multiphase Flow, 14, 565-574. https://doi.org/10.1016/0301-9322(88)90058-4

[39] Bellani, G., Nole, M.A. and Variano, E.A. (2013) Turbulence Modulation by Large Ellipsoidal Particles: Concentration Effects. Acta Mechanica, 224, 2291-2299. https://doi.org/10.1007/s00707-013-0925-z

[40] Mandø, M. and Rosendahl, L. (2010) On the Motion of Non-Spherical Particles at High Reynolds Number. Powder Technology, 202, 1-13.

https://doi.org/10.1016/j.powtec.2010.05.001

[41] Ljus, C., Johansson, B. and Almstedt, A.-E. (2002) Turbulence Modification by Particles in a Horizontal Pipe Flow. International Journal of Multiphase Flow, 28, 10751090. https://doi.org/10.1016/S0301-9322(02)00020-4

[42] Li, J., Wang, H., Liu, Z., Liu, Y., Han, H. and Zheng, C. (2010) Experimental Investigation on Turbulence Modulation in the Boundary Layer of a Horizontal Particle-Laden Channel Flow with Relative Low Mass Loading Ratios. The 6th International Symposium on Multiphase Flow, Heat Mass Transfer and Energy Conversion, 2010, 436-441.

\section{Submit or recommend next manuscript to SCIRP and we will provide best service for you:}

Accepting pre-submission inquiries through Email, Facebook, LinkedIn, Twitter, etc.

A wide selection of journals (inclusive of 9 subjects, more than 200 journals)

Providing 24-hour high-quality service

User-friendly online submission system

Fair and swift peer-review system

Efficient typesetting and proofreading procedure

Display of the result of downloads and visits, as well as the number of cited articles

Maximum dissemination of your research work

Submit your manuscript at: http://papersubmission.scirp.org/

Or contact epe@scirp.org 